\title{
Propuesta para estudiar las profesiones de la información: una contribución al análisis desde el proceso de profesionalización de la bibliotecología
}

Ruth Helena Vallejo Sierra*

Artículo recibido:

9 de marzo de 2019

Artículo aceptado:

4 de septiembre de 2019

Artículo de investigación

\section{RESUMEN}

La sociedad va creando profesiones de acuerdo con sus necesidades, este proceso implica características de tipo técnico y social que permiten conocer cómo se han desarrollado, sus objetivos y entender las realidades de su actuar presente. Este artículo presenta una propuesta para estudiar la profesión bibliotecológica en el contexto colombiano, a partir de un diseño metodológico de carácter mixto, cualitativo y cuantitativo, utilizando el método Delphi, cuya finalidad fue poner de manifiesto convergencias de opinión y llegar a ciertos consensos en torno a la conformación de categorías y subcategorías para estructurar una propuesta de estudio de la profesión. A partir de ello, se construyeron

* Universidad Distrital Francisco José de Caldas, Bogotá, Colombia rhvallejos@udistrital.edu.co INVESTIGACIÓN BIBLIOTECOLÓGICA, vol.34, núm. 83, abril/junio, 2020, México, ISSN: 2448-8321 pp. 85-101 
tres grandes categorías: la caracterización profesional, la identidad profesional y el ejercicio profesional, que son la base para construir el esquema sugerido para hacer estudios integrales de la bibliotecología y la archivística de acuerdo a los contextos de cada territorio.

Palabras clave: Profesión; Bibliotecología; Archivística; Estudios de Profesiones

Proposal to study librarianship and archives studies: a contribution to the analysis from the professionalization process

Rutb Elena Vallejo Sierra

\section{Abstract}

Society creates professions in accordance with its own requirements, a process that implies technical and social characteristics which allow to know how these careers have been developed and cope with the realities of their present. This article presents a proposal to study the library and archival profession within the Colombian context, departing from a mixed methodical design, qualitative and quantitative, using the Delphi method, whose aim pretends to reveal convergence of opinions and attainment of consensuses around the formation of categories and subcategories in order to structure a proposal of study of this career. Departing from there, three mayor categories were created: professional characterization, professional identity, and professional practice which are the basis for building the suggested outline to create thorough studies of library and information science according to the context of every region.

Keywords: Profession; Librarianship; Archives Studies; Professional Studies

\section{INTRODUCCIÓN}

$\mathrm{H}^{\circ}$ oy la bibliotecología y la archivística como profesiones deben dar respuesta a varias situaciones: la discusión teórica sobre su consolidación como ciencias, el papel que ejercerán en el acceso a la información, cómo 
apoyarán la apropiación social de las bibliotecas y archivos, su contribución al logro de los objetivos de desarrollo sostenible y al desarrollo social y cultural, donde sea indiscutible el aporte que hacen para mejorar la calidad de vida de la comunidad y de las organizaciones. Por consiguiente, las profesiones deben reflexionar sobre su función en la sociedad y cómo están comunicando este mensaje, para hacer evidente el beneficio de su misma existencia en un contexto o de lo contrario reformularse y proyectarse.

En este sentido, existen muchas respuestas que dar en cuanto a lo que esperan los demás de lo que debe ser un profesional de la bibliotecología y la archivística: ¿cuál es el papel que se le atribuye?, ¿son la bibliotecología y la archivística lo que esperan los demás, o tienen la capacidad de cambiar el papel y ajustarse a los cambios sociales? Es preciso retomar lo que afirma Abundis (2013: 907) frente a los factores que influyen en la valoración que hace la sociedad de una profesión:

Dependiendo de la utilidad que esa profesión representa en un determinado momento del desarrollo de la sociedad, cultura o grupo, considerando además la importancia de los servicios que prestan los profesionales a la sociedad, las necesidades que satisfacen y el grado en que lo logran. Asimismo, es importante considerar que las actividades profesionales resiginifican $[$ sic $]$ la realidad social porque la transforman, le atribuyen nuevos significados, definen sus dinámicas e interpretan las situaciones, identifican los problemas que ésta tiene y proponen alternativas para resolverlos, poniendo en juego las habilidades de los profesionales.

De esta manera, realizar estudios integrales de una profesión permite legitimar sus objetivos, validar su existencia y sentido social, además de hacer visible su función e influencia en la sociedad. Pero estos análisis deben centrarse en un entorno específico, pues cada sociedad valora y reconoce de manera diferente una profesión de acuerdo con sus necesidades y desarrollo. Por ende, este documento presenta la construcción de una propuesta para estudiar estas profesiones a partir de la investigación realizada por Vallejo (2014) sobre la profesión bibliotecológica en Colombia, como un aporte para que se puedan realizar estudios en los diferentes países, tomando en cuenta la ausencia de estudios técnicos y sociales que evidencien el proceso de profesionalización y que permitan comprender los avances, los retrocesos y el papel de la comunidad profesional en ellos.

Es necesario precisar que en algunos países existe un contexto institucional, legislativo y profesional común para la bibliotecología y la archivística; 
en Colombia, sin embargo, hay diferencias entre ambas disciplinas: ${ }^{1}$ existen leyes de ejercicio profesional diferentes, ley 11 de 1979 para el caso de la bibliotecología y ley 1409 para el caso de la archivística, con sus códigos de ética independientes; algunas escuelas independientes de formación, como es el caso de la Universidad Distrital y la Universidad de Antioquia que tienen programas exclusivos en el campo de la archivística, y asociaciones profesionales autónomas por cada profesión: Colegio Colombiano de Bibliotecología Ascolbi y Colegio Colombiano de Archivística.

Este documento presenta inicialmente una contextualización de las profesiones en la sociedad, con los antecedentes que se encontraron en la revisión teórica sobre la bibliotecología y archivística como profesiones; posteriormente se presenta el desarrollo metodológico de la investigación realizada a partir del método Delphi y finalmente una propuesta para realizar futuros estudios a partir de múltiples dimensiones.

\section{LAS PROFESIONES Y LA SOCIEDAD}

Las profesiones han surgido a medida que ha avanzado la sociedad. Tal como lo explican Landeros-Olvera y Amador-Gómez (2018: 37), “el concepto de profesión traspasa el mero ámbito escolar, puesto que su desarrollo ha estado ligado en forma permanente a la evolución de las sociedades, en donde se ha asumido características que le permiten ser una disciplina de servicio a la estructura social a la que pertenece", la cual le asigna un papel y una autonomía en el contexto que esta profesión se desarrolle.

La sociología ha desarrollado una serie de criterios para revisar el proceso mediante el cual una ocupación se convierte en profesión. Estos criterios son descritos desde diversas teorías y escuelas, a partir de las cuales es posible, por ejemplo, confirmar el grado de profesionalización de acuerdo con el cumplimiento de los indicadores definidos. En ese sentido, algunos autores los han aplicado argumentando sobre su alcance, o descalificándolos en la medida que advierten el nivel en que las profesiones los han logrado.

Por otro lado, vale la pena destacar la función social positiva desempeñada por las profesiones, como la que describe Quintanilha (2019: 13):

En primer lugar, en el sentido de que se basan en sistemas de reglas y valores como los de la racionalidad, conocimiento, meritocracia, igualdad de oportunidades, bienestar social y justicia; en segundo lugar, porque representan lógicas de

1 De acuerdo con la revisión teórica, los factores mencionados desde la escuela clásica del estudio de las profesiones marcarían la existencia de dos profesiones distintas para el caso colombiano. 
motivación altruista y acción orientada hacia el desarrollo del conocimiento, servicios de mejor calidad y defensa del interés público; y en tercer lugar, para citar a Rodríguez (2012, p. 28), en el sentido que se considera que "las profesiones constituyen una forma alternativa de organización del trabajo y la autoridad, basada en el conocimiento y no sobre características individuales o recursos heredados".

Para analizar los rasgos fundamentales de una profesión, Eslava Castañeda (2004) propone tener en cuenta un proceso importante para el orden social al involucrar una dinámica de especialización de saberes y habilidades que luego se orientarán al mantenimiento del sistema social. En este sentido, analizando las características de tipo técnico y social que definen la naturaleza de este campo del conocimiento, el estudio para comprender la bibliotecología y la archivística como profesiones debe abarcar múltiples dimensiones, no sólo cómo se desarrolla, organiza y mantiene su autonomía profesional, sino también la organización social y su relación con las profesiones y su conocimiento, el marco de socialización y las dinámicas de interacción social.

De la socialización profesional desde la perspectiva de Dubar (2000), pensada como el proceso de adquisición de la identidad donde se apropia el conjunto de elementos que diferencian y asimilan al profesional y a la sociedad, se deduce que a través de ella se entiende lo que somos, lo que sabemos, lo que hacemos. De esta manera, la identidad es aquello que con independencia del campo de acción, del nivel de intervención y del contexto en que alguien se ubique, le permite reconocerse como un profesional. Esto en el entendido de que la identidad sólo se consolida a través de la interacción social, a partir de las relaciones que establecen la profesión y el profesional en los diversos espacios sociales en que éste realiza su ejercicio, con lo cual se valida como profesión, se asume su misión y aceptación social.

En ese orden de ideas, es previsible suponer que se requieren muchas dimensiones para comprender una profesión, su surgimiento, continuidad, su capacidad de adaptarse a los cambios y su poder de innovación. A este respecto, López Yepes (2011) se refiere a transformaciones que evolucionan en tres aspectos imprescindibles en su desarrollo: la capacidad de generar conocimiento, la formación de los profesionales y sus posibles nuevas funciones, y la transferencia de los nuevos conocimientos a los sectores productivos y empresariales. De hecho, la estructura de las unidades de información y los sectores de actividad donde la información es necesaria están cada vez más diversificados. Surge entonces la pregunta de si la bibliotecología y la archivística tendrán la capacidad de adaptarse a los cambios que esta sociedad les demanda y las implicaciones que esto les acarrea, en términos que la sociedad las necesite o que sean solamente aceptadas como profesiones por 
una parte de la sociedad, o incluso que se conviertan en una alerta hacia su desprofesionalización.

En esta mirada de los profesionales de la información, Cunha (2013) sostiene que los cambios tecnológicos y estructurales de la sociedad de la información están obligando a los profesionales de la información a redefinir su lugar en el mercado de trabajo. Al mismo tiempo, las funciones informativas se han tornado más complejas y específicas. Esta tendencia a la especificidad no ha resultado en el fortalecimiento de las profesiones clásicas de la información (bibliotecarios, documentalistas y archiveros), pero ha abierto posibilidades a especialistas de otras disciplinas.

En el campo específico de la archivística, Martín-Pozuelo (2009: 17) afirma:

A nadie se le escapa que todas las profesiones hoy están sufriendo un cambio profundo en su naturaleza y en sus funciones, algunas además en su consideración social. Mientras unas alcanzan cotas de prestigio otras se denostan progresivamente. Hablamos de nuevos intelectuales, nuevos investigadores, nuevos administradores, nuevos ejecutivos, en fin, ¿podemos hablar también de nuevos archiveros?, ¿Qué cambio real se está produciendo?

\section{Antecedentes y estudios relacionados}

La revisión documental expuso que las profesiones que han sido más estudiadas en su proceso de profesionalización han sido enfermería, trabajo social y educación. No obstante, en su estudio sobre las profesiones en España, Martín-Moreno y De Miguel (1982) mencionan que este tipo de investigaciones se desconocen, y algunas concretas no cuentan con el fundamento sociológico, por lo que sugieren realizar indagaciones como:

1) Estudios comparados de dos o más profesiones en un mismo ámbito (geográfico, científico, organizativo). Esto resultaría de gran interés para conocer la realidad de las profesiones bibliotecológica y archivística en Iberoamérica o incluso las diferencias formativas y de titulaciones.

2) Monografías sobre profesiones de las que no se conoce nada. En este caso cabrían la bibliotecología y la archivística, pero en el marco de las teorías sociológicas.

3) Estudios del comportamiento habitual de algunas profesiones, de sus sistemas de trabajo y de la relación con sus usuarios, tales como las representaciones sociales que la comunidad tiene de los archivos, las bibliotecas y sus profesionales. 
4) Investigaciones de seguimiento, para ver el cambio experimentado en un determinado círculo profesional, por lo menos en dos momentos. Puede ser de gran interés en el caso colombiano revisar qué impacto ha tenido la ley de ejercicio profesional de la archivística diferente al de la bibliotecología.

A partir de los tipos de investigaciones sugeridas por Martín-Moreno y De Miguel (1982), los estudios de la profesión están ajustados a los tipos dos y tres en el caso de la bibliotecología y la archivística. En la búsqueda bibliográfica no se hallaron investigaciones sobre estudios comparativos o de seguimiento. En cuanto a las monografías sobre profesiones dentro de los enfoques sociológicos, los estudios especializados sobre la profesión archivística son prácticamente inexistentes; en el caso de la bibliotecología vale la pena mencionar los trabajos de Goode (1966) y de Winter (1983), en los cuales se realizan análisis sobre el proceso de profesionalización para considerar un punto importante: si la bibliotecología es o no una profesión y/o el grado de profesionalización que ha alcanzado.

En el caso de los estudios centrados en el comportamiento habitual de la profesión, fue posible encontrar análisis conjuntos de la bibliotecología y la archivística, así como individuales para cada una de las profesiones, enfocados en aspectos específicos como:

- La imagen de la profesión y del profesional, en su gran mayoría desde las representaciones sociales.

- Los aspectos relativos a currículos, perfiles, competencias y en general lo relacionado con la formación profesional.

- Los estudios del mercado laboral, que son los que más se encuentran en el sistema de búsqueda.

- El futuro de la profesión, especialmente en torno al impacto de las tecnologías.

Para el caso de investigaciones de las profesiones en un contexto específico, Melo de Souza (2010) se enfoca en la archivística para Brasil, que a partir del estudio del comportamiento de tres variables (formación, colectivos profesionales y mercado laboral) describe la realidad de los archiveros e identifica su visibilidad en relación a los archiveros con formación académica. Los resultados apuntaron a nuevos rumbos y nuevas direcciones de los graduados del curso ya que la praxis archivística ha extrapolado los límites de la Ley $\mathrm{n}^{\circ}$ 6.546, de 1978, que regula la profesión y que considera que el archivero crea nuevos espacios de trabajo y se incorpora en las investigaciones. 
Entre las investigaciones que estudian la bibliotecología en un contexto específico destacan las realizadas para Italia, África, Israel y Brasil. En 1997 Jennifer Marshall realizó el estudio "La bibliotecología como una profesión en Italia”, en el que concluye que realmente no puede decirse que el papel del bibliotecario en aquel país sea considerado una profesión: no existe un reconocimiento del ejercicio profesional por parte del gobierno y no se reconocen los títulos expedidos por las universidades, con lo cual no se estimula el estudio de la bibliotecología. Asimismo, a pesar de la representatividad de la asociación profesional, no se ha logrado avanzar en el proceso de profesionalización.

En 2006, en "La educación de los bibliotecarios y científicos de la información y su visibilidad, identidad y reconocimiento social en Brasil”, Souza llevó a cabo una investigación con enfoque cualitativo de carácter exploratorio en aspectos relacionados con la identidad profesional y la visibilidad, así como reflexiones sobre el reconocimiento social de la profesión, con base en la literatura brasileña en bibliotecología y ciencias de la información. En cuanto a la parte académica, deben ser tratados como proyectos político-pedagógicos bajo un doble enfoque: la preparación de personal para actuar en un entorno profesional que requiere habilidades técnicas y de gestión (formación de posgrado en bibliotecología) y preparación de personal para trabajar en un entorno profesional que requiere habilidades de investigación (la formación de posgrado en ciencias de la información).

Como antecedente en Colombia, vale la pena mencionar la investigación "La bibliotecología como alternativa profesional", que realizó Vallejo Sierra en 2008, cuyo objetivo era comprender las representaciones que los profesionales, estudiantes de bibliotecología y estudiantes de colegio tenían con respecto a la profesión. Encontró que no hay una sola concepción en los posibles aspirantes, no se evidencia como una profesión y se corresponde exclusivamente con el trabajo en una biblioteca (servicio de referencia). Las creencias se centran en considerarla un oficio y no una profesión, además de que no se necesita ninguna formación para pasar libros; persiste la imagen tradicional del bibliotecario si bien se percibe la aparición de nuevos estereotipos que asocian exclusivamente a las bibliotecas y solamente con el formato libros. El ejercicio profesional está caracterizado por un bajo estatus, reducido prestigio y condiciones laborales inadecuadas, la oferta de trabajo es el factor decisivo para ingresar y no es una opción profesional para los aspirantes.

Estas investigaciones sirvieron de punto de partida para estructurar la propuesta, considerar el planteamiento general de la investigación en torno a revisar cómo se han alcanzado cada una de las dimensiones y servir de guía para concretar las categorías de estudio. Esta ausencia de indagaciones integrales puede ser la causa de haber intentado soluciones parciales o reducidas. 
Por ello es imprescindible analizar el proceso de profesionalización como un todo y cómo repercute en su naturaleza, socialización y relaciones con la sociedad, para luego vislumbrar de qué modo el análisis contribuye a que la sociedad esté dispuesta a aceptar y dar a la bibliotecología y a la archivística su posición como profesiones, con todas las ventajas que ello conlleva.

\section{Овjetivo}

Comprender el proceso de profesionalización, para ello se desarrolló primero un estudio referente a la bibliotecología en Colombia dentro de los referentes sociológicos. Esto requirió construir una propuesta de estudio de la profesión, ya que la complejidad de la indagación requiere no sólo un cuidadoso análisis teórico, sino también la comprensión y significación de las ideas y conocimientos de los grupos sociales involucrados en la construcción y desarrollo de la profesión: estudiantes, profesionales, asociaciones profesionales, entidades que contratan sus servicios e instituciones que los forman.

\section{Metodología}

El estudio tiene un enfoque de carácter mixto: cualitativo y cuantitativo, que inició con una revisión documental, para luego desarrollar el método Delphi. Con las fuentes bibliográficas consultadas se buscó contar con los recursos que fundamentaran el proceso investigativo y así atender las siguientes necesidades:

- Contextualizar el proyecto de investigación en la realidad social de las profesiones, desde el enfoque de las escuelas sociológicas que plantean diferentes miradas a su estudio. Esto fue clave para la estructuración del trabajo y el esquema deductivo de las categorías y subcategorías de investigación.

- Establecer marcos teóricos de los elementos del proceso de profesionalización y los referentes desde la bibliotecología como profesión en general, así como los modelos de estudios desarrollados en otros países.

A partir del planteamiento teórico, se comprendió el proceso de profesionalización y sus implicaciones, para entender los mecanismos por los cuales una ocupación asume los atributos de una profesión, el dinamismo del 
proceso, su secuencialidad y que no existía un modelo único para estudiar una profesión que pudiera ser utilizado en esta investigación.

Esto permitió asumir que durante la profesionalización surgen cambios internos estructurales en la profesión y en las relaciones de los profesionales con la sociedad en general. Así, era indispensable construir una propuesta de estudio basado en las premisas obtenidas. Este texto recoge el proceso llevado a cabo con los expertos a través del método Delphi, que como lo explica López Gómez (2018) se usa para investigaciones que tratan de evaluar, identificar, estimar el uso, obtener consenso, predecir, definir, dimensionar, establecer líneas de trabajo, explorar, desarrollar y comprender. Se consideró adecuado para el grupo de expertos con base en lo siguiente:

- Ofrece un camino conveniente para estudiar temas tan complejos y dinámicos como la profesionalización de la bibliotecología en Colombia. Por la razón misma de su metodología (procedimiento), ayuda a obtener un conocimiento sobre una realidad social de la que no se cuenta con mucha información.

- Permitió convocar a los distintos expertos del país, a quienes era difícil concentrar por su dispersión geográfica y la dificultad para reunirlos.

- Disminuyó la influencia que podían tener algunos de los expertos sobre los demás integrantes del grupo, facilitando con esto que cada uno de ellos expresara sus opiniones abiertamente. Los participantes no tuvieron conocimiento de quiénes formaban parte del proyecto, pues sólo interactuaron con la investigadora.

- El objetivo del método es tratar de llegar a un consenso fiable entre las opiniones de los presidentes de asociaciones y miembros de escuelas de bibliotecología, a través de los diferentes cuestionarios anónimos.

El primer cuestionario de expertos se diseñó sobre la base de los criterios de profesionalización y las categorías definidas por cada uno de ellos, de manera que se permitía que cada uno dentro de su conocimiento expresara su visión del sentido de la bibliotecología como profesión. Este cuestionario se basa en la revisión teórica previa y plantea preguntas abiertas, de manera que se puedan conocer las diversas ideas propuestas, en forma muy amplia. Las preguntas se organizaron en tres bloques: caracterización profesional, identidad profesional y ejercicio profesional. Dada la complejidad de las preguntas se dio libertad a los participantes de expresar abiertamente sus opiniones sobre estos temas. El segundo cuestionario se diseñó para evaluar los resultados del primer cuestionario a través de la escala Likert. 
La definición de la población a estudiar se realizó de acuerdo con la relevancia de sus perfiles: experiencia profesional, vinculación con la profesión, en la academia o las asociaciones, y trayectoria en la profesión. Esto permitió recoger su punto de vista como especialistas en el tema, generar hipótesis más precisas y ser una fuente teórica de gran relevancia. Así se conformó el grupo de expertos formado por presidentes de las asociaciones profesionales, los cuales incluyen tanto a los organismos creados para agrupar a los miembros de la profesión y representarlos ante la sociedad, como a las agremiaciones fundadas por los egresados de las escuelas de bibliotecología; además, profesores y directores de escuelas profesionales. La selección de los participantes se realizó a nivel nacional.

Por tratarse de encontrar acuerdos el método Delphi sugiere el envío de una serie de cuestionarios, el primero se remite a 67 expertos con preguntas abiertas basadas en el sentido de la bibliotecología como profesión a partir de la revisión teórica; estos resultados fueron sistematizados y categorizados. Para la construcción del segundo cuestionario se establecieron preguntas cerradas y se solicitó la valoración cuantitativa de cada cualidad, teniendo en cuenta el tipo de pregunta cerrada y de selección múltiple a través de la escala Likert, para luego jerarquizar los temas y el grado de discrepancias y consensos. Del primer cuestionario se recibieron 26 respuestas. El segundo cuestionario se envió sólo a este grupo, y se logró obtener respuesta de 22 encuestados.

Con estos resultados se analiza tanto el consenso general de las preguntas como la estabilidad de la opinión de los expertos. En primer lugar, se construyó un total de cinco intervalos, los cuales asignan el grado de consenso que se tiene: muy bajo, bajo, medio, alto, muy alto. Posteriormente, con estos grados se clasificaron los acuerdos más altos obtenidos en cada pregunta. En la literatura que se consultó sobre investigaciones que aplican el método Delphi se observó que el mínimo grado aceptable es alto (igual o superior a 60 \%).

En cuanto a la estabilidad de la opinión de los expertos, se calculó el porcentaje de preguntas en las que cada experto mantiene la misma respuesta, tanto en la primera fase como en la segunda. A partir de estos resultados se obtiene una "estabilidad promedio" (que es la media de la estabilidad presentada por las respuestas de los expertos). Este cálculo se realizó para cada una de las dimensiones en que se dividió el cuestionario y para el cuestionario total. En promedio, los expertos mantienen sus respuestas en $34 \%$ de las preguntas (aproximadamente tres de ocho preguntas) en la dimensión 1, en $58 \%$ de las preguntas (aproximadamente cinco de nueve preguntas) en la dimensión 2, y en la dimensión 3 en 37 \% de las preguntas (aproximadamente cuatro de 10 preguntas). El resultado promedio total de estabilidad 
es $43.3 \%$, lo que quiere decir que en promedio los expertos mantienen sus respuestas en aproximadamente 12 de 27 preguntas.

\section{Resultados}

Los hallazgos nos indicaron que para tener una visión integral, completa y holística de una profesión no basta con establecer su naturaleza, entendida ésta desde las propiedades que la caracterizan. Se requiere además que los miembros del colectivo se apropien de los elementos identitarios y que el entorno los asimile, en un proceso de socialización, como factores que posibilitan la categorización, ya que la sociedad le irá otorgando a esa profesión una serie de privilegios o beneficios de acuerdo con la necesidad y el servicio que ésta le represente y las relaciones que vaya construyendo. Así se configura el reconocimiento profesional que la sociedad le concederá.

En ese sentido, se encontró que para construir una propuesta para estudiar una profesión debería partirse de tres ejes fundamentales:

- Establecer la naturaleza de la profesión, para revisar el conjunto de propiedades que la caracterizan como tal, lo que implica examinar el tránsito que ha recorrido en búsqueda de su sentido social, representado en los hechos históricos que describen el nivel de evolución y avance, su institucionalidad, la aportación específica que ofrece, la organización social del ejercicio profesional, la autonomía y los valores deontológicos que enmarcan el proceso de profesionalización.

- Describir el proceso de socialización, donde se apropia el conjunto de elementos que diferencian y asimilan el profesional y la sociedad, en el cual se interiorizan los valores y conocimientos de la profesión por medio de los diferentes significantes que construyen la identidad profesional, como su base de conocimiento, los procesos formativos, las titulaciones profesionales, la elección profesional, sus ámbitos, funciones y los retos de la profesión.

- Analizar las relaciones de la profesión con la sociedad, los elementos que involucran la proyección y el actuar profesional y su incidencia en las dinámicas sociales, que se manifiestan en el ejercicio profesional y que proyectan su apropiación social, que enmarca la valoración, el reconocimiento y prestigio social, la perspectiva de género, la imagen y mercado laboral. 
Las categorías y subcategorías definidas para el análisis de las profesiones se describen en la Tabla 1.

\begin{tabular}{|c|c|}
\hline Categorías & Subcategorías \\
\hline \multirow{5}{*}{$\begin{array}{c}\text { Caracterización } \\
\text { profesional }\end{array}$} & Hechos o eventos históricos que configuran el nacimiento de la profesión \\
\hline & Institucionalidad: regulaciones, políticas y legislación \\
\hline & Función social y servicio a la sociedad \\
\hline & Valores deontológicos y código de ética \\
\hline & Organización social del ejercicio profesional \\
\hline \multirow{6}{*}{$\begin{array}{l}\text { Identidad } \\
\text { profesional }\end{array}$} & Aprendizaje formal \\
\hline & Cuerpo de conocimientos, saber particular \\
\hline & Titulaciones profesionales \\
\hline & Elección profesional, vocación profesional \\
\hline & Ámbitos y funciones \\
\hline & Retos y oportunidades \\
\hline \multirow{5}{*}{$\begin{array}{l}\text { Ejercicio } \\
\text { profesional }\end{array}$} & Reconocimiento y valoración social \\
\hline & Prestigio profesional \\
\hline & Género \\
\hline & Imagen social \\
\hline & Mercado laboral \\
\hline
\end{tabular}

Tabla 1. Categorías de análisis para las profesiones Fuente: elaboración propia

Ahora bien, las subcategorías consideradas para la caracterización profesional reflejan los elementos básicos que han definido los modelos de profesionalización tradicionales, de las diversas escuelas sociológicas, las cuales son:

- El contexto actual en que está inmersa la profesión y su incidencia.

- Los hechos que han enmarcado el desarrollo, desde sus inicios hasta los logros actuales.

- Los significados asociados a la bibliotecología y la archivística como oficios, profesiones, disciplinas o ciencias.

- La legislación, las políticas, las normas y las regulaciones existentes que han dado sentido y legitimidad social a la bibliotecología y la archivística.

- La función que cumplen la bibliotecología y la archivística en la sociedad, para mostrar su utilidad y el espacio donde se necesitan. 
- Las asociaciones profesionales como representantes del grupo profesional.

- Las alcances del código de ética.

De acuerdo con los hallazgos, la identidad profesional del bibliotecólogo y el archivista se debe estudiar a partir de:

- El conocimiento único y especializado de la bibliotecología y la archivística.

- La formación profesionalque reciben los bibliotecólogos y los archivistas colombianos, con sus fortalezas y oportunidades de mejoramiento.

- Los conocimientos, habilidades y actitudes que deben potenciarse en el profesional.

- Las esferas de desempeño y las funciones que hoy tiene el ejercicio profesional.

- Las denominaciones de bibliotecólogo y de archivista como mecanismo de acreditación del título profesional.

- Las motivacionesque existen para la elección de la bibliotecología y la archivística como profesiones.

- Los retos que deben enfrentar la bibliotecología y la archivística y las posibilidades de adaptarse a esos cambios.

La visión que proyectan la bibliotecología y la archivística como resultado del ejercicio profesional puede ser analizada a través de los siguientes aspectos:

- El reconocimiento social que se les ha otorgado y los factores que han contribuido a esto.

- El aporte que hacen a la sociedad, considerado desde su valoración social.

- El prestigio profesional, medido desde la visión de otras profesiones y los factores que originan las escalas de valoración.

- Los problemas que enfrentan el bibliotecólogo y el archivista en su ejercicio profesional en todos los contextos en que éste se da.

- La imagen del profesional en la sociedad.

- El mercado laboral profesional desde sus condiciones, trayectorias e inserción.

- Las iniciativas planteadas para beneficiar la proyección y el actuar profesional. 


\section{Conclusión}

Esta propuesta se relaciona con las condiciones fundamentales que conciben a la bibliotecología y la archivística como profesiones, así como su ser, saber y hacer que configuran la identidad profesional y lo que se proyecta de ésta. Con ello se puede tener una base de conocimiento que facilite el alcance de sus objetivos como profesiones, el mejoramiento de su proyección a la sociedad y recuperar su influencia social; es decir, que a partir de conocer la realidad profesional se pueden descubrir sus principales fortalezas y oportunidades de mejoramiento para reconstruir un proceso de profesionalización que lidere una comunicación con la sociedad en todos los sentidos y grupos sociales.

Se pretendió realizar una propuesta para estudiar las profesiones no desde el exterior, como se acostumbra en los trabajos de estereotipos, imágenes y mercado laboral, sino desde el interior para poder realizar una autocrítica. Esto debido a que sólo desde un autodiagnóstico es posible que los bibliotecólogos y los archivistas conozcan el estado real de sus profesiones, desde una visión holística, y desde allí reflexionar sobre los aspectos en que deben concentrarse para lograr un espacio profesional más representativo.

Es posible que las profesiones tengan muchos modelos para ser estudiadas. El planteamiento que se hizo fue producto de una reflexión profunda desde lo teórico para pensar en un abordaje diferente, ya que hay estudios sobre una o varias de las categorías, pero ninguno incluye un conjunto articulado de categorías para dar una visión más completa de las profesiones. Por otra parte, los cambios permanentes y las dificultades que implica la profesionalización hacen que sea preciso plantear una perspectiva abierta, más orientativa que descriptiva del proceso. Pero al final lo importante no es realizar un inventario del conjunto de rasgos distintivos, sino delimitar entre éstos los que son utilizados por los profesionales para afirmar y sostener sus relaciones sociales y cómo son apropiados por todos.

La configuración y aplicación de esta propuesta se hizo inicialmente para el caso de estudio de la bibliotecología, y nos debe conducir a iniciar el estudio de la archivística que nos dará la visión completa de las profesiones de la información en Colombia, entendiendo que en otros contextos esta división no es aplicable, con lo cual será necesario realizar ajustes metodológicos y de categorías para analizar las dos profesiones de manera conjunta.

El uso del método Delphi presentó muchos retos, especialmente porque los expertos en la mayor parte de los casos no tuvieron estabilidad en sus respuestas y tardaron mucho tiempo en responder. Aunque la obtención de consenso es el objetivo central del método, es posible que el alto nivel de dispersión se derive de la complejidad del tema y de la situación actual de las 
profesiones en el país, en cuanto a divergencias en la formación, en las titulaciones, en el tiempo de enseñanza, etc. Sin embargo, se lograron sistematizar, categorizar y jerarquizar los temas. Por supuesto, este conjunto de criterios sirven para examinar los atributos que constituyen una profesión y contrastar cómo van sufriendo cambios a través del tiempo o cuáles de ellos no han completado su desarrollo e incluso cuáles son inexistentes o han desaparecido. No obstante, debe entenderse como un proceso, sin secuencialidad, con logros en algunas categorías, retrocesos o ningún avance en otros. Asimismo, las categorías están afectadas por un entorno diverso y cambiante, con presencia de sucesos intrínsecos y extrínsecos a la propia categoría o a su relación con las demás.

Por supuesto, registrar el proceso social mediante el cual la bibliotecología y la archivística se han ido profesionalizando en cualquier país permitirá comprender la apropiación que la sociedad ha hecho de ellas y su incidencia entre quienes diseñan las políticas públicas, toman las decisiones vocacionales, los planes de estudio, los programas de comunicación, la visibilidad y reconocimiento, y por lo tanto, aportar los elementos necesarios para edificar una nueva lectura, reconstruir las dinámicas sociales, fortalecer su desarrollo en el país y contribuir a su redimensionamiento. Adicionalmente nos permitiría hacer estudios comparativos, conocer las buenas prácticas de otros por países y conocer la realidad bibliotecológica y archivística latinoamericana desde una mirada integral.

\section{REFERENCIAS}

Abundis, F. 2013. "El papel de las profesiones dentro de la dinamica de la vida social". Trabajo presentado en 1er. Congreso Internacional de Investigación Educativa, Nuevo León, México, 28, 29 y 30 de agosto.

Cunha Vieira Da, M. 2013. "Las profesiones de la información: un escenario de cambios”. Ciencias de la Información 44 (1). https://www.redalyc.org/html/1814/181428544001/

Dubar, C. 2000. La socialisation: construcciòn de identités sociales et professionelles. París: Armand Colin.

Eslava Castañeda, J. 2004. Buscando el reconocimiento profesional: la salud pública en Colombia, en la primera mitad del siglo XX. Bogotá: Universidad Nacional de Colombia.

Goode, W. 1966. "The librarian: from occupation to profession”, en Professionalization, editado por Howard M. Vollmer y Donld L. Mills, 34-45. New Jersey, Estados Unidos: Prentice Hall.

Landeros-Olvera, E. y Amador-Gómez, M. 2018. "Enfermería: de la ocupación a la profesión, de la profesión a la ciencia”. Saludy Administración 5 (14): 35-42. 
López Gómez, E. 2018. “El método Delphi en la investigación actual en educación: una revisión teórica y metodológica”. Educación XX1 21 (1): 17-40.

https://doi.org/10.5944/educXX1.20169

López Yepes, J. 2011. "Algunas repercusiones de la sociedad de la información en la gestación de nuevas profesiones informativas”. Signo y Pensamiento 30 (59). http://www.scielo.org.co/scielo.php?script=sci_arttext\&pi$\mathrm{d}=$ S0120-48232011000200001\&lng $=$ en\&nrm $=$ iso

Marshall, J. 1997. "Librarianship as a profession in Italy". Journal of Librarianship and Information Science 29 (1): 29-37. https://doi.org/10.1177/096100069702900104

Martín-Moreno, J. y De Miguel, A. 1982. Sociología de las profesiones en España. Madrid: Centro de Investigaciones Sociológicas.

Martín-Pozuelo, M. Paz. 2009. "Los entornos y documentos electrónicos ¿Efecto Edison para la formación archivística". Ponto de Acesso 3 (1): 6-33.

Melo de Souza, K. 2010.”Análisis y evolución del panorama laboral del archivero en Brasil: el Poder Legislativo Federal en escena”. Tesis de Maestría, Universidad Carlos III de Madrid, Departamento de Biblioteconomía y Documentación. https://e-archivo.uc3m.es/bitstream/handle/10016/9156/TESIS_KATIA_Isabe1li\%20de\%20Bethania\%20Melo\%20de\%20Souza.pdf

Quintanilha, T. L. 2019. “Journalists' professional self-representations: a Portuguese perspective based on the contribution made by the sociology of professions". Journalism. Post-print. https://repositorio.iscte-iul.pt/bitstream/10071/17251/1/Accepted\%20version.pdf

Rodríguez Ávila, N. 2008. Manual de sociología de las profesiones. Barcelona: Universidad de Barcelona.

Souza, F. das Chagas de. 2006. "A formação acadêmica de bibliotecários e cientistas da informação e sua visibilidade, identidade e reconhecimento social no Brasil”. Informação \& Sociedade: Estudos 16 (1): 32-46. http://fundamentos1.files.wordpress.com/2009/11/souza-a-formacao-academica-de-bibliotecarios.pdf

Vallejo Sierra, R. 2008. "La bibliotecología como alternativa profesional”. Trabajo de grado para obtener la suficiencia investigadora, Universidad de Salamanca, Facultad de Traducción y Documentación.

Vallejo Sierra, R. 2014. "La bibliotecología como profesión en Colombia”. Tesis de doctorado, Universidad de Salamanca, Facultad de Traducción y Biblioteconomía. https://universoabierto.org/2016/01/26/la-bibliotecologia-como-profesion-en-colombia/

Winter, Michael F. 1983. "The Professionalization of Librarianship”. Occasional Paper 160:3-46.

Para citar este texto:

Vallejo Sierra, Ruth Helena. 2020. "Propuesta para estudiar las profesiones de la información: una contribución al análisis desde el proceso de profesionalización de la bibliotecología”. Investigación Bibliotecológica: archivonomía, bibliotecología e información 34 (83): 85-101. http://dx.doi.org/10.22201/iibi.24488321xe.2020.83.58092 